\title{
ELEMENTARY SOLUTIONS FOR A MODEL BOLTZMANN EQUATION IN ONE DIMENSION AND THE CONNECTION TO GROSSLY DETERMINED SOLUTIONS
}

\author{
THOMAS E CARTY
}

\begin{abstract}
The Fourier-transformed version of the time dependent slip-flow model Boltzmann equation associated with the linearized BGK model is solved in order to determine the solution's asymptotics. The ultimate goal of this paper is to demonstrate that there exists a robust set of solutions to this model Boltzmann equation that possess a special property that was conjectured by Truesdell and Muncaster: that solutions decay to a subclass of the solution set uniquely determined by the initial mass density of the gas called the grossly determined solutions. First we determine the spectrum and eigendistributions of the associated homogeneous equation. Then, using Case's method of elementary solutions, we find analytic time-dependent solutions to the model Boltzmann equation for initial data with a specialized compact support condition under the Fourier transform. In doing so, we show that the spectrum separates the solutions into two distinct parts: one that behaves as a set of transient solutions and the other limiting to a stable subclass of solutions. Thus, we demonstrate that for gas flows with this specialized initial density condition, in time all gas flows for the one dimensional model Boltzmann equation act as grossly determined solutions.
\end{abstract}

\section{INTRODUCTION}

We consider a simplification of the linearized Boltzmann equation in one dimension. Let $x \in \mathbb{R}$ represent the position of a molecule and let $v \in \mathbb{R}$ be the velocity of that molecule. We consider the model of fluid motion dictated by

$$
\frac{\partial f}{\partial t}(t, x, v)+v \frac{\partial f}{\partial x}(t, x, v)=-f(t, x, v)+\int_{-\infty}^{\infty} \phi(w) f(t, x, w) d w
$$

where $\phi(w)$ is the probability density function $\phi(v)=e^{-v^{2}} / \sqrt{\pi}$ and the unknown function $f(t, x, v)$ represents the molecular density function of a monotomic gas. In the classical Boltzmann theory, the right hand side of (1.1) is interpreted as the collision operator - dictating the behavior of the gas under inter-molecular collisions. In [2], the three dimensional version $\left(\mathbf{x}, \mathbf{v} \in \mathbb{R}^{3}\right)$ of the model Boltzmann (1.1) is derived and the collisions operator is demonstrated to possess the conservation of mass condition under molecular collision. For the purely one dimensional equation (1.1), an alternative derivation (known as the slip-flow problem) was constructed by Cercignani when splitting the one dimensional (in $x$ ) BGK equation [1]. (See [5], [11], [9], or [18], for a more modern discussion.)

2010 Mathematics Subject Classification. 35Q35, 76P99, 46F12.

Key words and phrases. linearized Boltzmann equation, rarified slip flow equation, asymptotics of elementary solutions, grossly determined solutions.

The author acknowledges support from National Science Foundation grant DMS 08-38434 "EMSW21-MCTP: Research Experience for Graduate Students" and from the Caterpillar Fellowship Grant at Bradley University. 
In $[28$, Ch. XXIII], C. Truesdell and R. G. Muncaster remark that - no matter which model of gas flow you begin with - the ultimate goal is the same: determine the first few moments of the gas. Namely, the density, velocity and temperature fields of the gas. They then note that many of the known exact solutions of Boltzmann's equation - such as those solutions derived from Hilbert's iteration (see [10, pg. 316] or [28, Ch. XXII]), or the Chapman and Enskog procedure (see [20, pg. 86]) - shared the property that the solution class could be represented as being dependent on one (or more) of the gas's physical properties (moments). This led them to define the concept of a grossly determined solution: a solution which is determined at any given instant by the gross conditions (mass density, velocity, temperature) of the gas at that time. In their epilogue, the authors suggest that these concepts may lead to a new way forward for finding unifying solutions to the Boltzmann equation:

(1) In general, can we determine a set of conservation laws that define the gross field properties?

(2) Can we use these conservation laws to determine the class of grossly determined solutions to the problem?

(3) If one could find the class of general solutions, can we show that the general solutions evolve asymptotically in time to the class of grossly determined solutions?

In addition to finding a new, richer class of solutions to the Boltzmann equation (a microscopic/atomic level model of gas flow), the class of grossly determined solutions would now be in terms akin to the solutions of the Navier-Stokes equations (a macroscopic/gross fields model of gas dynamics).

The main result in [2] was to demonstrate that a class of grossly determined solutions exists to (1.1) that depends solely on the density field of the gas. In doing so, the first two conjectures were shown true for the model Boltzmann (1.1). In [2], we were led to consider initial data such that the zeroth moment (i.e. mass density) would possess compact support on $|\xi|<\sqrt{\pi}$ under the Fourier transform in the spacial variable. Then it was shown that given the density of the gas at any moment of time, one can determine the density function $\rho(t, x)$ of the gas for all time and subsequently a solution $f(t, x, v)$ to (1.1), which itself is a function of $\rho(t, x)$. It should be noted that a grossly determined solution based upon the zeroth moment alone is the best that can be expected for the model equation (1.1). As opposed to the full Boltzmann equation, the collision operator $C(f):=-f(t, x, v)+\int_{\mathbb{R}} \phi(w) f(t, x, w) d w$ only possesses a conservation of mass condition. In the reduction of the model, conservation of momentum and energy have been lost in the collision operator. Thus, we don't expect to find grossly determined solutions based upon the higher moments of velocity and energy. The goal of this paper is to demonstrate the third item, that when using the same initial conditions required in [2], elementary solutions (solutions determined via separation of variables) to (1.1) exist that evolve asymptotically in time to the class of grossly determined solutions found in [2]. To this end, there are three main components of this paper: construction of the solution candidate via spectral methods, proving that the candidate solution is complete and unique, and demonstrating the desired decay condition.

Solutions to (1.1) under the Laplace transform were first indicated by Cercignani [6] (additionally see Chapter VII of [8] or [18] (pg. 289-292)) using Case's method of elementary solutions [4]. While in many ways emulating the same technique as Cercignani, there are some distinct differences in this current work. For one, we will be constructing solutions under the Fourier transform. This is due to the fact that the construction of the grossly 
determined solutions obtained in [2] was done under the Fourier transform. As a result, the spectrum and eigensolutions are of a different form than in Cericignani's work. The spectral representation of the solution (in both Cercignani's work and this work) results in an integral equation of the third kind where the integral is over $\mathbb{R}$. However, in this work we will show there is an additional portion of the continuous spectrum for the operator. In fact, it is exactly this new real-valued line segment in the spectrum that corresponds to the subclass of grossly determined solutions and guarantees the asymptotic behavior in Truesdell and Muncaster's third conjecture. We will also show that the familiar portion of the spectrum, corresponding to the infinite line resulting in the integral equation of the third kind, behaves as a set of transient solutions. In the end, we show that for the gas flows described by our elementary solutions for the model Boltzmann (1.1) act as grossly determined solutions.

The majority of the applications of Case's method are to linear transport equations and result in integral equations over the domain $[-1,1]$ where special care needs to be taken at the end points. (See $[19,22,23]$.) For application of elementary solutions to model Boltzmann equations, the boundary conditions are often either overlooked (see [6,9]) or addressed by a change of variables transformation so the integral is over $[-1,1]$ (see $[12,13])$ and solved using more modern integral equation methods (see [15]). We desire a clean connection between grossly determined solutions and the general solution class. To this end, we appeal to a result of Gakhov [17] that allows us to solve the associated Riemann-Hilbert problem from the natural integral equation that arises from integrating over the infinite spectrum. In the end, we will have an exact solution to the model Boltzmann (1.1) that is dependent upon initial data.

\section{Associated Spectral Problem}

We begin by taking the Fourier transform of equation (1.1) in the spatial variable. This yields the transformed PIDE

$$
\frac{\partial \hat{f}}{\partial t}(t, \xi, v)=-v i \xi \hat{f}(t, \xi, v)-\hat{f}(t, \xi, v)+\int_{\mathbb{R}} \phi(w) \hat{f}(t, \xi, w) d w
$$

and we write this as

$$
\frac{\partial \hat{f}}{\partial t}(t, \xi, v)=L(\hat{f})(t, \xi, v)
$$

where the operator $L$ is defined to be

$$
L(g)(\xi, v):=-\xi v i g(\xi, v)-g(\xi, v)+\int_{\mathbb{R}} \phi(w) g(\xi, w) d w .
$$

We need to consider an appropriate class of functions on which $L$ will operate. In the classical theory on the Boltzmann equation it is traditional to work with a function space defined by a weighted $L_{2}$ norm. Doing so imbues the operator on the right-hand side of the Boltzmann equation (the collision operator) with the desired properties of being selfadjoint and negative definite. (See [14].) In [2], the linear operator $C(g):=-g(\xi, v)+$ $\int_{\mathbb{R}} \phi(w) g(\xi, w) d w$ is shown to be, for each fixed $\xi$, self-adjoint and semi-negative definite on the function space $\mathscr{F}_{v}$ defined to be the class of functions such that

$$
\|f(v)\|_{2, \phi}^{2}=\int_{\mathbb{R}}|f(v)|^{2} \phi(v) d v<\infty \text { where } \phi(v)=e^{-v^{2}} / \sqrt{\pi} .
$$


Note that $L$ can be written in terms of the collision operator,

$$
L(g)=-\xi v i g+C(g) .
$$

We want to guarantee that repeated applications of $L$ to a function from our chosen function space will remain in our function space. To this end, we require that our functions are Schwartz class in both the spatial and velocity variables. Thus we define $\mathscr{F}$ such that

$$
\|f(\xi, v)\|_{2, \phi}^{2}=\int_{\mathbb{R}^{2}}|f(\xi, v)|^{2} \phi(v) d v d \xi<\infty
$$

and such that $\lim _{\xi, v \rightarrow \pm \infty} \xi^{n} v^{m} f(\xi, v)=0$ for all $n, m \in \mathbb{N}$. By construction, the operator $L$ maps functions from $\mathscr{F}$ to $\mathscr{F}$ and repeated applications of the operator $L$ to a function $f(\xi, v) \in \mathscr{F}$ will remain in the space of functions.

\section{The Resolvent Operator}

In order to determine the potential spectrum of $L$, we begin by identifying the resolvent operator. Upon constructing the spectral decomposition, we are likely to find that we need even more that just operating over the functions space $\mathscr{F}$. However, at this time we can formally find $(L-\lambda)^{-1}$ and use its construction to hint at where the spectrum of $L$ must lie.

Proposition 1. Let $h \in \mathscr{F}$ and $\lambda \in \mathbb{C}$ and consider the equation $(L-\lambda) g=h$. Then the formal inverse of $(L-\lambda)$ is defined by

$$
(L-\lambda)^{-1} h(\xi, v):=\frac{-1}{1+\lambda+\xi v i}\left(h(\xi, v)+\frac{\int_{\mathbb{R}} \frac{h(\xi, v) \phi(v) d v}{1+\lambda+\xi v i}}{1-\int_{\mathbb{R}} \frac{\phi(v) d v}{1+\lambda+\xi v i}}\right) .
$$

Proof. We begin with $(L-\lambda) g=h$ and formally solve for $g$ :

$$
-v i \xi g(\xi, v)-g(\xi, v)+\int_{\mathbb{R}} \phi(w) g(\xi, w) d w-\lambda g(\xi, v)=h(\xi, v) .
$$

Provided that $1+\lambda+\xi v i \neq 0$, we find that

$$
g(\xi, v)=-\frac{1}{1+\lambda+\xi v i}\left(h(\xi, v)-\int_{\mathbb{R}} \phi(w) g(\xi, w) d w\right) .
$$

We now seek an expression for $\int_{R} \phi(w) g(\xi, w) d w$, independent of $g(\xi, v)$, that we can use to substitute into the last equation. Multiplying by $\phi(v)$ and integrating with respect to $v$ yields

$$
-\int_{\mathbb{R}} \phi(v) g(\xi, v) d v=\int_{\mathbb{R}} \frac{\phi(v) h(\xi, v) d v}{1+\lambda+\xi v i}-\left(\int_{\mathbb{R}} \phi(w) g(\xi, w) d w\right)\left(\int_{\mathbb{R}} \frac{\phi(v) d v}{1+\lambda+\xi v i}\right) .
$$

Now, provided that $1-\int_{\mathbb{R}} \frac{\phi(v) d v}{1+\lambda+\xi v i} \neq 0$, we obtain the following expression:

$$
\int_{\mathbb{R}} \phi(w) g(\xi, w) d w=-\frac{\int_{\mathbb{R}} \frac{h(\xi, v) \phi(v) d v}{1+\lambda+\xi v i}}{\left(1-\int_{\mathbb{R}} \frac{\phi(v) d v}{1+\lambda+\xi v i}\right)} .
$$


The computation in the previous proof suggests where the spectrum may be located. We look for spectral values to occur for $\lambda$ such that either

$$
1-\int_{\mathbb{R}} \frac{\phi(v) d v}{1+\lambda+\xi v i}=0 \text { or } 1+\lambda+\xi v i=0 .
$$

Upon determining where the spectrum must lie, we will return to the formal inverse of $(L-\lambda)$ and demonstrate that we have captured the resolvent set.

Remark. In this instance, the computation of (3.1) was straightforward enough to do directly. The same could have been accomplished via the theory of rank-one perturbations of self-adjoint operators. From this viewpoint, the spectrum of a rank-one perturbation can be determined by the spectrum of the unperturbed operator and information about the perturbation determinant $d=1-\int_{\mathbb{R}} \frac{\phi(v) d v}{1+\lambda+\xi v i}$. (See [27] or [25]). Here Equation (3.1) is closely related to the Aronszjan-Krein formula. More specifically, Equation (3.1) is exactly Equation (2.3) in the lecture notes of Liaw and Treil [25] using the operator $L$.

\section{The Spectral Decomposition}

We search for a basis (set of generalized eigenfunctions) $B(\xi, v)$ such that $L(B)=\lambda B$. It is important to note that in this spectral problem, we interpret it as a spectral problem in $v$ dependent on a parameter $\xi$. We are not computing the spectral problem in 2-dimensions simultaneously. Using the definition of $L$, the eigenvalue problem is

$$
-i \xi v B(\xi, v)-B(\xi, v)+\int_{\mathbb{R}} \phi(w) B(\xi, w) d w=\lambda B(\xi, v)
$$

We then have a recursive representation of the basis function

$$
B(\xi, v)=\frac{\int_{\mathbb{R}} \phi(w) B(\xi, w) d w}{1+\lambda+\xi v i} .
$$

Define

$$
b(\xi):=\int_{\mathbb{R}} \phi(w) B(\xi, w) d w .
$$

Now (4.2) becomes

$$
B(\xi, v)=\frac{b(\xi)}{1+\lambda+\xi v i} .
$$

Multiplying by $\phi(v)$ and integrating over the velocity space yields

$$
b(\xi)=b(\xi) \int_{\mathbb{R}} \frac{\phi(v) d v}{1+\lambda+\xi v i} .
$$

The above computation results in a constraint equation for $\lambda$ dependent on variable $\xi$.

Proposition 2. Let $B(\xi, v)$ satisfy the spectral equation $L(B)=\lambda B$ and let

$$
b(\xi)=\int_{\mathbb{R}} \phi(w) B(\xi, w) d w .
$$

For all $\xi$ for which $b(\xi) \neq 0, \lambda$ must satisfy the constraint

$$
\int_{\mathbb{R}} \frac{\phi(v) d v}{1+\lambda+\xi v i}=1 .
$$




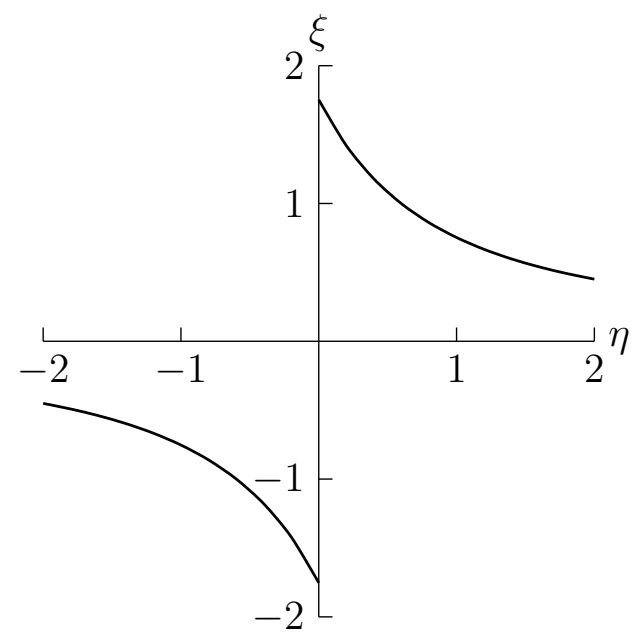

FiguRE 1. the graph of $\xi=\Xi(\eta)$

\subsection{The Spectrum: The Real-Valued Portion.}

\subsubsection{The interval $(-1,0)$.}

Proposition 3. The interval $(-1,0) \subseteq \mathbb{R}$ is part of the spectrum.

Proof. Assume $\lambda$ is real-valued. Begin with the left-hand side of constraint equation (4.5).

$$
\begin{aligned}
\int_{\mathbb{R}} \frac{\phi(v) d v}{1+\lambda+\xi v i} & =\int_{\mathbb{R}} \frac{\phi(v)[(1+\lambda)-(\xi v) i] d v}{(1+\lambda)^{2}+(\xi v)^{2}} \\
& =(1+\lambda) \int_{\mathbb{R}} \frac{\phi(v) d v}{(1+\lambda)^{2}+(\xi v)^{2}}-\xi i \int_{\mathbb{R}} \frac{v \phi(v) d v}{(1+\lambda)^{2}+(\xi v)^{2}} \\
& =(1+\lambda) \int_{\mathbb{R}} \frac{\phi(v) d v}{(1+\lambda)^{2}+(\xi v)^{2}} \quad \text { (since the right-hand integrand is odd in } v \text {.) }
\end{aligned}
$$

Therefore, when $\lambda$ is real, the constraint equation for $\lambda$ reduces to

$$
\int_{\mathbb{R}} \frac{(1+\lambda) \phi(v) d v}{(1+\lambda)^{2}+(\xi v)^{2}}=1
$$

Note that this equation requires that $\lambda \neq-1$.

In [3], the odd invertible function $\Xi: \mathbb{R} \backslash 0 \rightarrow(-\sqrt{\pi}, 0) \cup(0, \sqrt{\pi})$ defined

$$
\Xi(\eta):=\int_{\mathbb{R}} \frac{\eta \phi(v) d v}{\eta^{2}+v^{2}}
$$

was analyzed. (See Figure 1.)

Provided $\xi \neq 0$, we see that (4.6) can be rewritten as

$$
\begin{gathered}
\int_{\mathbb{R}} \frac{\left(\frac{1+\lambda}{\xi}\right) \phi(v) d v}{\left(\frac{1+\lambda}{\xi}\right)^{2}+v^{2}}=\xi, \text { or } \\
\Xi\left(\frac{1+\lambda}{\xi}\right)=\xi .
\end{gathered}
$$




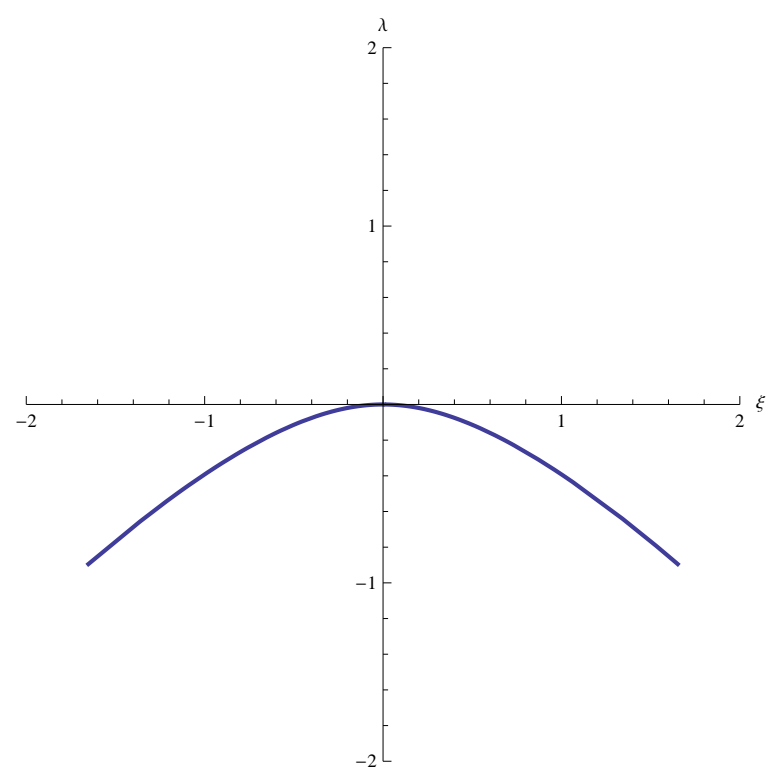

FIGURE 2. the graph of $\lambda=\Lambda(\xi)$

This suggests that we should seek to use the the inverse of $\Xi(\eta)$ to rewrite $\lambda$ in terms of $\eta$, and hence $\xi$. Let $\eta(\xi)=\Xi^{-1}(\xi)$. Using (4.6), we equate $\eta=\frac{1+\lambda}{\xi}$ and find a parametric representation for $\lambda=\Lambda(\xi)$ where $\Lambda(\xi):=-1+\xi \eta(\xi)$, the graph of which is Figure 2 .

To formally analyse the range of values $\lambda$ can take and still satisfy the identity (4.6), we look to the range of the function $\Lambda(\xi)$. Given that $\eta \rightarrow 0^{+}$, as $\xi \rightarrow(\sqrt{\pi})^{-}$, we see that $\lim _{\xi \rightarrow \sqrt{\pi}^{-}}(-1+\xi \eta(\xi))=-1$. To determine the behavior of $\lambda$ as $\xi$ goes to 0 , we examine the behavior of $\xi \eta(\xi)$. Here, it is easier to look at the inverse problem and examine $\Xi(\eta) \eta$. We have that

$$
\Xi(\eta) \eta=\left(\int_{\mathbb{R}} \frac{\eta \phi(v) d v}{\eta^{2}+v^{2}}\right) \eta=\int_{\mathbb{R}} \frac{\eta^{2} \phi(v) d v}{\eta^{2}+v^{2}} .
$$

Hence,

$$
\begin{aligned}
\lim _{\eta \rightarrow \infty} \Xi(\eta) \eta & =\lim _{\eta \rightarrow \infty} \int_{\mathbb{R}} \frac{\eta^{2} \phi(v) d v}{\eta^{2}+v^{2}}, \\
& =\lim _{\eta \rightarrow \infty} \int_{\mathbb{R}} \frac{\left(\eta^{2}+v^{2}-v^{2}\right) \phi(v) d v}{\eta^{2}+v^{2}}, \\
& =\int_{\mathbb{R}} \phi(v) d v-\lim _{\eta \rightarrow \infty} \int_{\mathbb{R}} \frac{v^{2} \phi(v) d v}{\eta^{2}+v^{2}}, \\
& =1-0 .
\end{aligned}
$$

Thus, $\lim _{\eta \rightarrow \infty}(-1+\Xi(\eta) \eta)=0$. This demonstrates that the real-valued portion of the spectrum is the interval $(-1,0)$.

Since $\eta(\xi)$ is an odd, invertible function, analysis of $-1+\Xi(\eta) \eta$ as $\eta \rightarrow 0^{-}$and $\eta \rightarrow-\infty$ results in the same portion of the real axis. 
4.1.2. Associated Eigendistributions. At this moment, we appear to have a collection of spectral values that are dependent upon the parameter $\xi$. We now will free $\lambda$ of its parametric dependence and construct functions akin to eigensolutions.

We know that $\lambda$ and $\xi$ are related by

$$
1-\int_{\mathbb{R}} \frac{\phi(v) d v}{1+\lambda+\xi v i}=0
$$

and this can be rewritten as $\xi=\Xi\left(\frac{1+\lambda}{\xi}\right)$. This means that $\lambda=-1+\xi \eta(\xi)=\Lambda(\xi)$ where we have a nice graph of $\Lambda$ in Figure 2. Thus, for any $\xi$ in the domain of $\Lambda$ :

$$
\int_{\mathbb{R}} \frac{\phi(v) d v}{1+\Lambda(\xi)+\xi v i}=1
$$

and (4.7) is not valid if $\lambda$ does not have the form $\Lambda(\xi)$ for some $\xi$.

We can see from the graph of $\Lambda$ that for each fixed value of $\lambda$ in the interval $(-1,0)$ there corresponds two values of $\xi$. In turn, we can identify these corresponding values of $\xi$ with $\lambda$. Let $E^{+}(\lambda)$ denote the inverse of $\Lambda(\xi)$ over the restricted domain $\xi \in(0, \sqrt{\pi})$ and let $E^{-}(\lambda)$ denote the inverse of $\Lambda(\xi)$ over $\xi \in(-\sqrt{\pi}, 0)$. In this notation, for each fixed $\lambda$ in $(-1,0)$, $E^{+}(\lambda)$ and $E^{-}(\lambda)$ are the corresponding values of $\xi$. Thus (4.8) can be rephrased in terms of $\lambda$ rather than $\xi$ as

$$
\int_{\mathbb{R}} \frac{\phi(v) d v}{1+\lambda+E^{+}(\lambda) v i}=1 \text { and } \int_{\mathbb{R}} \frac{\phi(v) d v}{1+\lambda+E^{-}(\lambda) v i}=1
$$

and (4.7) is not valid if $\xi$ does not have the form $E^{+}(\lambda)$ or $E^{-}(\lambda)$ for some $\lambda$ in $(-1,0)$.

We now use these facts and build eigendistributions. Let $B_{\lambda}$ be the basis function dependent on $\lambda$. Beginning with (4.2), the recursive definition of $B(\xi, v)$, we use the definition of $b_{\lambda}(\xi)$ to derive

$$
b_{\lambda}(\xi)\left(1-\int_{\mathbb{R}} \frac{\phi(v) d v}{1+\lambda+\xi v i}\right)=0 .
$$

From (4.9) we see that $b_{\lambda}(\xi)$ must vanish at all points $\xi$ other than $E^{+}(\lambda)$ and $E^{-}(\lambda)$. Additionally, the value of $b_{\lambda}(\xi)$ is arbitrary at these two points. This leads us to the two distributional solutions

$$
b_{\lambda}^{+}=\delta\left(\xi-E^{+}(\lambda)\right) \text { and } b_{\lambda}^{-}=\delta\left(\xi-E^{-}(\lambda)\right),
$$

where $\delta(x)$ denotes the Dirac delta distribution. In general, $b_{\lambda}$ will be an arbitrary superposition of these two distributions. By (4.2), we obtain two eigendistributions for each $\lambda$ in $(-1,0)$ :

$$
B_{\lambda}^{+}(\xi, v)=\frac{\delta\left(\xi-E^{+}(\lambda)\right)}{1+\lambda+\xi v i} \text { and } B_{\lambda}^{-}(\xi, v) \frac{\delta\left(\xi-E^{-}(\lambda)\right)}{1+\lambda+\xi v i}
$$

Our ultimate goal is to create a transform. That is, we will seek to integrate in $\lambda$ over the interval $(-1,0)$ an arbitrary superposition of the basis functions of the form

$$
C^{+}(\lambda, \xi) \frac{\delta\left(\xi-E^{+}(\lambda)\right)}{1+\lambda+\xi v i}+C^{-}(\lambda, \xi) \frac{\delta\left(\xi-E^{-}(\lambda)\right)}{1+\lambda+\xi v i} .
$$

As posed, the integration requires a change of variables so that the delta distributions are of the form $\delta(\lambda-\Gamma)$ where $\Gamma$ is independent of $\lambda$. Note that all the action of $\delta\left(\xi-E^{+}(\lambda)\right)$ occurs at $\xi=E^{+}(\lambda)$. Since $\Lambda(\xi)$ is the inverse of $E^{+}(\lambda)$, we can equivalently say that all of the action 
of $\delta\left(\xi-E^{+}(\lambda)\right)$ occurs at $\Lambda(\xi)=\lambda$. This suggests that we redefine $b_{\lambda}(\xi)=\delta(\lambda-\Lambda(\xi))$. Note that we are not claiming that $\delta\left(\xi-E^{+}(\lambda)\right)=\delta(\lambda-\Lambda(\xi))$. In fact, the change of variables would require the Jacobian $\frac{1}{\left|\Lambda^{\prime}\left(E^{+}(\lambda)\right)\right|}$. However, since the Jacobian is now purely a function of $\lambda$, it can be subsumed by the arbitrary coefficient function. Therefore we can define $b_{\lambda}^{+}(\xi)=\delta(\lambda-\Lambda(\xi))$. Since $\Lambda(\xi)$ is also the inverse of $E^{-}(\lambda)$, the same argument concludes that $b_{\lambda}^{-}(\xi)=\delta(\lambda-\Lambda(\xi))$. In essence, we obtain just one solution $b_{\lambda}(\xi)=\delta(\lambda-\Lambda(\xi))$ and the one eigendistribution

$$
B_{\lambda}(\xi, v)=\frac{\delta(\lambda-\Lambda(\xi))}{1+\lambda+\xi v i} .
$$

A somewhat simpler way to arrive at the same conclusion is to think of (4.10) in a different way. Rather than viewing this as a problem for a function of $\xi$ depending parametrically on $\lambda$, we can view it as a problem of solving for a function of $\lambda$ depending parametrically on $\xi$. Given our initial calculations, we conclude immediately that $b_{\lambda}(\xi)$ will be some multiple of $\delta(\lambda-\Lambda(\xi))$ and this leads immediately to (4.13).

Theorem 4. Define $L$ as in (2.1). Then the distribution $B_{\lambda}$ defined by (4.13) satisfies the equation $L\left(B_{\lambda}\right)=\lambda B_{\lambda}$ for each $\lambda \in(-1,0)$.

Proof. Notice that

$$
\begin{aligned}
L\left(B_{\lambda}\right)(\xi, v) & =L\left(\frac{\delta(\lambda-\Lambda(\xi))}{1+\lambda+\xi v i}\right) \\
& =-\xi v i\left(\frac{\delta(\lambda-\Lambda(\xi))}{1+\lambda+\xi v i}\right)-\left(\frac{\delta(\lambda-\Lambda(\xi))}{1+\lambda+\xi v i}\right)+\int_{\mathbb{R}} \frac{\phi(w) \delta(\lambda-\Lambda(\xi)) d w}{1+\lambda+\xi w i} \\
& =(-1-\xi v i)\left(\frac{\delta(\lambda-\Lambda(\xi))}{1+\lambda+\xi v i}\right)+\delta(\lambda-\Lambda(\xi)) \int_{\mathbb{R}} \frac{\phi(w) d w}{1+\lambda+\xi w i} .
\end{aligned}
$$

The last integral was analysed in the proof of Proposition 3. Moreover, in the derivation of $B_{\lambda}$, it was shown that when $\lambda=\Lambda(\xi)$, the integral is identically 1 . Hence,

$$
\begin{aligned}
L\left(B_{\lambda}\right)(\xi, v) & =(-1-\xi v i)\left(\frac{\delta(\lambda-\Lambda(\xi))}{1+\lambda+\xi v i}\right)+\delta(\lambda-\Lambda(\xi)) \\
& =\lambda\left(\frac{\delta(\lambda-\Lambda(\xi))}{1+\lambda+\xi v i}\right) \\
& =\lambda B_{\lambda}(\xi, v) .
\end{aligned}
$$

\subsection{The Spectrum: The Complex-Valued Portion.}

4.2.1. The line $\boldsymbol{\ell}:=-1+\alpha i$. The other divisibility condition required in the derivation of the resolvent operator (3.1) is that $1+\lambda+\xi v i \neq 0$. This suggest that we should consider spectral values of the form $\lambda=-1-\xi v i$. Since $\xi$ and $v$ are free variables over $\mathbb{R}$, this continuum of points is more concisely written $\lambda=-1+\alpha i, \alpha \in \mathbb{R}$. We define $\boldsymbol{\ell}$ to be this line in the complex plane. 
4.2.2. Associated Eigendistributions. We begin again with (4.1) and let $\lambda \in \boldsymbol{\ell}$. Then (4.2), the recursive form of $B(\xi, v)$, becomes

$$
B(\xi, v)=\frac{\int_{\mathbb{R}} \phi(w) B(\xi, w) d w}{(\alpha+\xi v) i} .
$$

Previously, we converted this equation to an equation in $b(\xi)$ and made use of the properties of the delta distribution. This time we don't have that luxury. Multiplying (4.14) by $\phi(v)$ and integrating over $v$-space yields

$$
b(\xi)\left(1-\int_{\mathbb{R}} \frac{d v}{(\alpha+\xi v) i}\right)=0 .
$$

The only solution to this equation is $b(\xi)=0$.

That said, our previous work indicated that we should be searching for a distributional basis function. Mimicking the technique suggested by Case [4] and Cercignani [6,7], we append to (4.14) the weighted Dirac mass whose action occurs at the singularity caused by $\alpha+\xi v$. That is,

$$
B(\xi, v)=\frac{\int_{\mathbb{R}} \phi(w) B(\xi, w) d w}{(\alpha+\xi v) i}+K(\xi) \delta(\xi v+\alpha) .
$$

where $K(\xi)$ is a function that will allow this basis candidate to satisfy the definition of $b(\xi)$.

Using the definition of $b(\xi)$, we solve for $K(\xi)$ explicitly.

$$
\begin{aligned}
b(\xi) & =\int_{\mathbb{R}} \phi(w) B(\xi, w) d w \\
& =b(\xi)\left(\int_{\mathbb{R}} \frac{\phi(w) d w}{(\alpha+\xi w) i}\right)+\int_{\mathbb{R}} \phi(w) K(\xi) \delta(\xi w+\alpha) d w .
\end{aligned}
$$

To make sense of the resultant integrals, we need to view them distributionally. In particular, the first integral is viewed as a Cauchy principal value integral (denoted p.v.). Hence,

$$
\begin{aligned}
b(\xi) & =b(\xi)\left(\text { p.v. } \int_{\mathbb{R}} \frac{\phi(w) d w}{(\alpha+\xi w) i}\right)+\int_{\mathbb{R}} \phi(w) K(\xi) \delta(\xi w+\alpha) d w \\
b(\xi) & \left.=b(\xi)\left(\text { p.v. } \int_{\mathbb{R}} \frac{\phi(w)}{i(\xi w+\alpha)} d w\right)+K(\xi) \int_{\mathbb{R}} \phi(\beta / \xi) \delta(\beta+\alpha) \frac{d \beta}{|\xi|} \quad \text { (where } \beta=\xi v\right) \\
b(\xi) & =b(\xi)\left(\text { p.v. } \int_{\mathbb{R}} \frac{\phi(w)}{i(\xi w+\alpha)} d w\right)+K(\xi) \frac{\phi(-\alpha / \xi)}{|\xi|} \\
K(\xi) & =\frac{|\xi| b(\xi)}{\phi(-\alpha / \xi)}\left(1-\text { p.v. } \int_{\mathbb{R}} \frac{\phi(w)}{i(\xi w+\alpha)} d w\right) .
\end{aligned}
$$

Using the derivation of $K(\xi), B(\xi, v)$ becomes

$$
B(\xi, v)=\frac{b(\xi)}{i(\xi v+\alpha)}+\frac{|\xi| b(\xi)}{\phi(-\alpha / \xi)}\left(1-\text { p.v. } \int_{\mathbb{R}} \frac{\phi(w)}{i(\xi w+\alpha)} d w\right) \delta(\xi v+\alpha) .
$$

Note that each term of $B(\xi, v)$ is being multiplied by $b(\xi)$. As we did before, we can let this be subsumed into the functional coefficient in the transform. (This has the added benefit of 
normalizing $B$ with respect to mass-density.) In other words, for this definition of $B(\xi, v)$, $b(\xi)=1$. Hence, for each $\lambda \in \boldsymbol{\ell}$, we define the associated eigendistribution

$$
B_{\lambda(\alpha)}(\xi, v)=\frac{1}{i(\xi v+\alpha)}+\frac{|\xi|}{\phi(-\alpha / \xi)}\left(1-\mathrm{p} \cdot \mathrm{v} \cdot \int_{\mathbb{R}} \frac{\phi(w)}{i(\xi w+\alpha)} d w\right) \delta(\xi v+\alpha) .
$$

Theorem 5. Define $L$ as in (2.1). Then for each $\lambda=-1+\alpha i \in \ell$, the distribution $B_{\lambda(\alpha)}$ (4.15) satisfies the equation $L\left(B_{\lambda}\right)=\lambda B_{\lambda}$.

Proof. This is a straightforward computation using the machinery built above:

$$
\begin{aligned}
L\left(B_{\lambda(\alpha)}\right) & =-\xi v i B_{\lambda(\alpha)}(\xi, v)-B_{\lambda(\alpha)}(\xi, v)+\int_{\mathbb{R}} \phi(w) B_{\lambda(\alpha)}(\xi, w) d w \\
& =-B_{\lambda(\alpha)}(\xi, v)-\xi v i B_{\lambda(\alpha)}(\xi, v)+1 \\
& =-B_{\lambda(\alpha)}(\xi, v)+\alpha i B_{\lambda(\alpha)}(\xi, v)-\alpha i B_{\lambda(\alpha)}(\xi, v)-\xi v i B_{\lambda(\alpha)}(\xi, v)+1 \\
& =(-1+\alpha i) B_{\lambda(\alpha)}(\xi, v)-(\alpha+\xi v) i B_{\lambda(\alpha)}(\xi, v)+1 \\
& =(-1+\alpha i) B_{\lambda(\alpha)}(\xi, v)+[-1-K(\xi)(\alpha+\xi v) \delta(\xi v+\alpha)]+1 \\
& =(-1+\alpha i) B_{\lambda(\alpha)}(\xi, v)+[-1+0]+1 \\
& =(-1+\alpha i) B_{\lambda(\alpha)}(\xi, v) .
\end{aligned}
$$

4.2.3. The Spectral Value $\lambda=0$. We have identified the line $\boldsymbol{\ell}$ and the interval $(-1,0)$ as belonging to the spectrum of $L$. For a "nice enough" operator, we would expect the complement of the resolvent set to be closed [24]. However, currently the union of our spectral pieces is not a closed set. We will close $\ell \cup(-1,0)$ by demonstrating that $\lambda=0$ is a spectral value.

Recall that by construction of the PIDE, $\int_{\mathbb{R}} \phi(v) d v=1$. By (4.6), when $\lambda=0$ we have

$$
\int_{\mathbb{R}} \frac{\phi(v) d v}{1+\xi^{2} v^{2}}=1
$$

It is clear that this identity will only hold when $\xi=0$. Now consider the graph of $\Lambda(\xi)$, Figure 2. This suggests that we should be able to redefine $\Lambda(\xi)$ continuously by including the point $(0,0)$. With this extension, equation (4.10) becomes

$$
b_{0}(\xi)\left[1-\int_{\mathbb{R}} \frac{\phi(v) d v}{1+\xi^{2} v^{2}}\right]=0
$$

and we get the distributional solution $b_{0}(\xi)=\delta(\lambda-\Lambda(0))$. Now the eigendistribution (4.13) can be extended to a basis function on the half-open interval $\lambda \in(-1,0]$. Hence, we have closed the spectrum.

4.3. The Resolvent Set. We now take a moment to show that we have in fact captured the spectrum of the operator $L$.

Theorem 6. Define $\mathbf{S}=\boldsymbol{\ell} \cup(-1,0]$. For $\lambda \in \mathbb{C} / \mathbf{S}$, the operator $(L-\lambda)^{-1}$ over $\mathscr{F}$ defined by (3.1) is bounded. In other words, $\mathbb{C} / \mathbf{S}$ is in the resolvent set of $L$. 
Proof. Fix $\lambda \in \mathbb{C} / \mathbf{S}$. Define $d$ to be the distance between the point $\lambda$ and the line $\boldsymbol{\ell}$; $d=\operatorname{dist}(\lambda, \boldsymbol{\ell})$. Additionally, for $\lambda \notin(-1,0]$, the proof of Proposition 3 shows that

$$
\int_{\mathbb{R}} \frac{(1+\lambda) \phi(v) d v}{(1+\lambda)^{2}+(\xi v)^{2}} \neq 1
$$

Hence, we define the constant $\gamma$ to be

$$
\gamma=1-\int_{\mathbb{R}} \frac{(1+\lambda) \phi(v) d v}{(1+\lambda)^{2}+(\xi v)^{2}} .
$$

Let $h$ be an arbitrary function from $\mathscr{F}$. Then,

$$
\begin{aligned}
\left\|(L-\lambda)^{-1} h(\xi, v)\right\|_{2, \phi} & =\left\|\frac{-1}{1+\lambda+\xi v i}\left(h(\xi, v)+\frac{\int_{\mathbb{R}} \frac{h(\xi, v) \phi(v) d v}{1+\lambda+\xi v i}}{1-\int_{\mathbb{R}} \frac{\phi(v) d v}{1+\lambda+\xi v i}}\right)\right\|_{2, \phi} \\
& \leq \frac{1}{d}\left(\|h(\xi, v)\|_{2, \phi}+\frac{1}{|\gamma|} \| \int_{\mathbb{R}} \frac{\left.h(\xi, v) \phi(v) d v \|_{1+\lambda+\xi v i}\right)}{1+\lambda}\right) \\
& \leq \frac{1}{d}\left(\|h(\xi, v)\|_{2, \phi}+\frac{1}{|\gamma| d}\left\|\int_{\mathbb{R}} h(\xi, v) \phi(v) d v\right\|_{2, \phi}\right) \\
& \leq \frac{1}{d}\left(1+\frac{1}{|\gamma| d}\right)\|h(\xi, v)\|_{2, \phi}
\end{aligned}
$$

Hence $\mathbf{S}$ is the continuous spectrum of $L$.

4.4. The Transform Candidate. Again letting $\mathbf{S}=\boldsymbol{\ell} \cup(-1,0]$, we seek to represent functions in the form

$$
\hat{f}(\xi, v)=\int_{\mathbf{S}} C(\lambda, \xi) B_{\lambda}(\xi, v) d \lambda
$$

By the preceding, we have

$$
\hat{f}(\xi, v)=\int_{(-1,0]} C(\lambda, \xi) B_{\lambda}(\xi, v) d \lambda+\int_{\ell} K(\lambda(\alpha), \xi) B_{\lambda(\alpha)}(\xi, v) d \alpha .
$$

We now examine these two integrals independently.

For $\lambda \in(-1,0]$, the integration is straightforward as our basis is a delta distribution in $\lambda$ :

$$
\begin{aligned}
\int_{(-1,0]} C(\lambda, \xi) B_{\lambda}(\xi, v) d \lambda & =\int_{(-1,0]} C(\lambda, \xi) \frac{\delta(\lambda-\Lambda(\xi))}{1+\lambda+\xi v i} d \lambda \\
& =\frac{C(\Lambda(\xi), \xi)}{1+\Lambda(\xi)+\xi v i} \\
& =\frac{C_{\Lambda}(\xi)}{1+\Lambda(\xi)+\xi v i}
\end{aligned}
$$

where $C_{\Lambda}(\xi):=C(\Lambda(\xi), \xi)$. 
For $\lambda \in \ell$, the integration is a little more technical. We already have a parametrization for $\boldsymbol{\ell}$ in terms of $\alpha$ and $B_{\lambda(\alpha)}$ in terms of the same parameter. So

$$
\begin{aligned}
\int_{\ell} K(\lambda(\alpha), \xi) & B_{\lambda(\alpha)}(\xi, v) d \alpha \\
& =\int_{\mathbb{R}} \widetilde{K}(\alpha, \xi) B_{\lambda(\alpha)}(\xi, v) d \alpha \quad \quad(\text { where } \widetilde{K}(\alpha, \xi):=K(-1+\alpha i, \xi)) \\
& =\int_{\mathbb{R}} \widetilde{K}(\alpha, \xi)\left[\frac{1}{i(\xi v+\alpha)}+\frac{|\xi|}{\phi(-\alpha / \xi)}\left(1-\mathrm{p} \cdot \mathrm{v} \cdot \int_{\mathbb{R}} \frac{\phi(w)}{i(\xi w+\alpha)} d w\right) \delta(\xi v+\alpha)\right] d \alpha \\
& =\text { p.v. } \int_{\mathbb{R}} \frac{\widetilde{K}(\alpha, \xi) d \alpha}{i(\xi v+\alpha)}+\frac{|\xi|}{\phi(v)}\left(1-\mathrm{p} \cdot \mathrm{v} \cdot \int_{\mathbb{R}} \frac{\phi(w)}{i(\xi w-\xi v)} d w\right) \widetilde{K}(-\xi v, \xi) .
\end{aligned}
$$

In the first integral, make the change of variables $\alpha=-\xi w$ to get

$$
\begin{aligned}
\int_{\ell} K(\lambda(\alpha), \xi) & B_{\lambda(\alpha)}(\xi, v) d \alpha \\
= & \text { p.v. } \int_{\mathbb{R}} \frac{\widetilde{K}(-\xi w, \xi)|\xi| d w}{i(\xi v-\xi w)}+\frac{|\xi|}{\phi(v)}\left(1-\mathrm{p} \cdot \mathrm{v} \cdot \int_{\mathbb{R}} \frac{\phi(w)}{i(\xi w-\xi v)} d w\right) \widetilde{K}(-\xi v, \xi) \\
& =-\mathrm{p} . \mathrm{v} \cdot \int_{\mathbb{R}} \frac{K_{\xi}(w) d w}{i(w-v)}+\frac{1}{\phi(v)}\left(\xi-\mathrm{p} \cdot \mathrm{v} \cdot \int_{\mathbb{R}} \frac{\phi(w)}{i(w-v)} d w\right) K_{\xi}(v)
\end{aligned}
$$

where $K_{\xi}(v):=\frac{|\xi|}{\xi} \widetilde{K}(-\xi v, \xi)$. Therefore, the integral transform associated with the spectral decomposition is

$$
\hat{f}(\xi, v)=\frac{C_{\Lambda}(\xi)}{1+\Lambda(\xi)+\xi v i}-\text { p.v. } \int_{\mathbb{R}} \frac{K_{\xi}(w) d w}{i(w-v)}+\frac{1}{\phi(v)}\left(\xi-\text { p.v. } \int_{\mathbb{R}} \frac{\phi(w)}{i(w-v)} d w\right) K_{\xi}(v)
$$

4.4.1. Applying the Operator to the Transform Candidate. Ultimately our goal is to use our spectral decomposition to solve for the general solution to $\hat{f}_{t}(t, \xi, v)=L \hat{f}$. It will be important to have a representation of $L \hat{f}$ in terms of the spectral coefficients $C_{\Lambda}(\xi)$ and $K_{\xi}(v)$. Since the computation of $L \hat{f}$ closely mirrors the computations above, we will prove the action of $L$ on our decomposition here.

Corollary 7. Let $L$ be defined by (2.1) and $B_{\lambda}$ defined by (4.13). Then

$$
L\left(\int_{(-1,0]} C(\lambda, \xi) B_{\lambda}(\xi, v) d \lambda\right)=\Lambda(\xi) \frac{C_{\Lambda}(\xi)}{1+\Lambda(\xi)+\xi v i}
$$


Proof.

$$
\begin{aligned}
L\left(\int_{(-1,0]} C(\lambda, \xi) B_{\lambda}(\xi, v) d \lambda\right) & =\int_{(-1,0]} C(\lambda, \xi) L\left(B_{\lambda}(\xi, v)\right) d \lambda \\
& \left.=\int_{(-1,0]} C(\lambda, \xi) \lambda B_{\lambda}(\xi, v)\right) d \lambda \quad \text { (by Theorem 4) } \\
& =\int_{(-1,0]} C(\lambda, \xi) \lambda \frac{\delta(\lambda-\Lambda(\xi))}{1+\lambda+\xi v i} d \lambda \\
& =\Lambda(\xi) \frac{C_{\Lambda}(\xi)}{1+\Lambda(\xi)+\xi v i} . \quad \text { (by computation similar to (4.13)) }
\end{aligned}
$$

Corollary 8. Let $L$ be defined by (2.1) and $B_{\lambda(\alpha)}$ be defined by (4.15). Then

$$
\begin{aligned}
& L\left(\int_{\ell} K(\lambda(\alpha), \xi) B_{\lambda(\alpha)}(\xi, v) d \alpha\right) \\
& \quad=- \text { p.v. } \int_{\mathbb{R}} \frac{(-1-\xi w i) K_{\xi}(w) d w}{i(w-v)}+\frac{1}{\phi(v)}\left(\xi-\mathrm{p} . \mathrm{v} \cdot \int_{\mathbb{R}} \frac{\phi(w)}{i(w-v)} d w\right)(-1-\xi v i) K_{\xi}(v) .
\end{aligned}
$$

Proof.

$$
\begin{aligned}
L\left(\int_{\ell} K(\lambda(\alpha), \xi) B_{\lambda(\alpha)}(\xi, v) d \alpha\right) & =\int_{\ell} K(\lambda(\alpha), \xi) L\left(B_{\lambda(\alpha)}(\xi, v)\right) d \lambda \\
& \left.=\int_{\ell} K(\lambda(\alpha), \xi)(-1+\alpha i) B_{\lambda(\alpha)}(\xi, v)\right) d \lambda \quad \text { ( by Theorem 5) } \\
& \left.\left.=-\int_{\ell} K(\lambda(\alpha), \xi) B_{\lambda(\alpha)}(\xi, v)\right) d \lambda+i \int_{\ell} K(\lambda(\alpha), \xi) \alpha B_{\lambda(\alpha)}(\xi, v)\right) d \lambda
\end{aligned}
$$

The first integral has been computed above as (4.18). Similarly,

$$
\begin{aligned}
i \int_{\ell} K(\lambda(\alpha), \xi) & \alpha B_{\lambda(\alpha)}(\xi, v) d \alpha \\
& =- \text { p.v. } \int_{\mathbb{R}} \frac{(\xi w i) K_{\xi}(w) d w}{i(w-v)}+\frac{1}{\phi(v)}\left(\xi-\text { p.v. } \int_{\mathbb{R}} \frac{\phi(w)}{i(w-v)} d w\right)(-\xi v i) K_{\xi}(v) .
\end{aligned}
$$

Summing these two integral yields the desired result.

4.5. Solving the Singular Integral Equation. We begin with the observation that the principal value integrals in (4.19) are multiples of the Hilbert transforms of $K_{\xi}(w)$ and $\phi(w)$, respectively. Numerically, the Hilbert transform of $e^{-x^{2}}$ is well understood in terms of the Dawson function $D(y)$ where $D(y)=e^{-y^{2}} \int_{0}^{y} e^{x^{2}} d x[29]$, see Figure 3. Specifically:

$$
\text { p.v. } \int_{\mathbb{R}} \frac{\phi(w)}{i(w-v)} d w=2 i D(v) .
$$




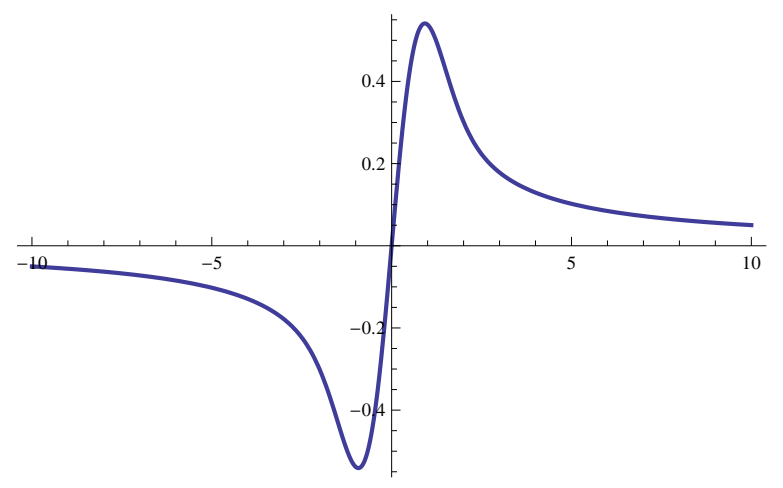

Figure 3. The graph of the Dawson function

We now manipulate (4.19) into the standard form for a Carleman type singular integral equation [15]:

$$
\begin{gathered}
\hat{f}(\xi, v)-\frac{C_{\Lambda}(\xi)}{1+\Lambda(\xi)+\xi v i}=- \text { p.v. } \int_{\mathbb{R}} \frac{K_{\xi}(w) d w}{i(w-v)}+\frac{1}{\phi(v)}(\xi-2 i D(v)) K_{\xi}(v), \\
\phi(v)\left(\hat{f}(\xi, v)-\frac{C_{\Lambda}(\xi)}{1+\Lambda(\xi)+\xi v i}\right)=(\xi-2 i D(v)) K_{\xi}(v)+\frac{-\pi \phi(v)}{\pi i} \text { p.v. } \int_{\mathbb{R}} \frac{K_{\xi}(w) d w}{w-v} .
\end{gathered}
$$

In order to simplify the following analysis, we make the following notational changes:

$$
F_{\xi}(v)=A_{\xi}(v) K_{\xi}(v)+\frac{B(v)}{\pi i} \mathrm{p} \cdot \mathrm{v} \cdot \int_{\mathbb{R}} \frac{K_{\xi}(w) d w}{w-v},
$$

where $A_{\xi}(v):=\xi-2 i D(v), B(v):=-\pi \phi(v)$, and

$$
F_{\xi}(v):=\phi(v)\left(\hat{f}(\xi, v)-\frac{C_{\Lambda}(\xi)}{1+\Lambda(\xi)+\xi v i}\right)
$$

By the definitions of $\phi(v)$ and the Dawson function $D(v)$, we know that $A_{\xi}(v)$ and $B(v)$ are Hölder continuous functions in $v$. We will require $\hat{f}(\xi, v)$ be such that $F_{\xi}(v)$ is Hölder continuous as well.

4.5.1. Reduction to an Associated Riemann Problem. Solving (4.21) requires converting the equation into its equivalent Riemann problem [16], [26]. Using the Sokhotski-Plemelj formulas, an equivalent representation of this problem is to seek a sectionally analytic function $\Phi_{\xi}(v)$ satisfying the boundary condition

$$
\Phi_{\xi}^{+}(v)=G_{\xi}(v) \Phi_{\xi}^{-}(v)+g_{\xi}(v)
$$

where $G_{\xi}(v)=\frac{A_{\xi}(v)-B(v)}{A_{\xi}(v)+B(v)}$ and $g_{\xi}(v)=\frac{F_{\xi}(v)}{A_{\xi}(v)+B(v)}$ on the real-axis in the complex plane. Solvability of this Riemann problem begins with the conditions that $G_{\xi}(v)$ and $g_{\xi}(v)$ are defined and non-vanishing on the real-line. Equivalently, we need $A_{\xi}(v)-B(v) \neq 0$ and $A_{\xi}(v)+B(v) \neq 0$. Note that $A_{\xi}(v)-B(v)=\xi+\pi \phi(v)-2 i D(v)$. On $(-\infty, \infty), D(v)$ vanishes only at $v=0$. When $v=0, A_{\xi}(0)-B(0)=\xi+\pi \phi(0)=\xi+\sqrt{\pi}$. Hence $A_{\xi}(v)-B(v)$ vanishes when $v=0$ and $\xi=-\sqrt{\pi}$. An equivalent computation shows that $A_{\xi}(v)+B(v)$ 
vanishes when $v=0$ and $\xi=\sqrt{\pi}$. For the remainder of this discussion, we will assume that the parameter $\xi \neq \pm \sqrt{\pi}$.

In the classical theory, the solution(s) to the Riemann problem

$$
\Phi^{+}(z)=G(z) \Phi^{-}(z)+g(z)
$$

are constructed for boundary problems with finite simple (often closed) boundary curve $\gamma$ in the complex plane. The representation of the problem's solution is dependent on the problem's index. Define

$$
\chi=\operatorname{Ind} G(z)=\frac{1}{2 \pi}[\arg G(z)]_{\gamma}
$$

where $[0]_{\gamma}$ denotes the increment of the expression in the brackets as the result of one traversal along $\gamma$. In other words, $\chi$ is the winding number of the image of the boundary curve $\gamma$ in $\mathbb{C}$ under the map $G(z)$. Let $X(z)$ be the solution to the associated homogeneous problem

$$
X^{+}(z)=G(z) X^{-}(z) .
$$

When $\chi \geq 0$, the general solution is a certain particular solution of (4.23) plus a summation of $\chi$ linearly independent solutions of the homogeneous problem. In particular, the solution is

$$
\Phi(z)=\frac{X(z)}{2 \pi i} \int_{\gamma} \frac{g(\tau)}{X^{+}(\tau)} \frac{d \tau}{\tau-z}+X(z) P_{\chi}(z)
$$

where $P_{\chi}(z)$ is a polynomial of degree $\chi$ with arbitrary coefficients. If we impose the additional constraint that $\Phi(z)$ decay to 0 at infinity, then the general solution has the same form except the polynomial must be of one degree less. Here the solution is

$$
\Phi(z)=\frac{X(z)}{2 \pi i} \int_{\gamma} \frac{g(\tau)}{X^{+}(\tau)} \frac{d \tau}{\tau-z}+X(z) P_{\chi-1}(z) .
$$

If $\chi<0$, the solution - with decay at infinity - takes the same form except now $P_{\chi}(z) \equiv 0$.

In Gakhov's untranslated Boundary Value Problems, 3rd edition [17, pg 191] we find the following result for Riemann problems with infinite boundary. For completeness, we have included the theorem in its entirety.

Theorem 9. ${ }^{1}$ The singular equation

$$
A(t) \phi(t)+\frac{B(t)}{\pi i} \int_{\mathbb{R}} \frac{\phi(\tau)}{\tau-t} d \tau=f(t)
$$

and the Riemann problem for the real line with the extra condition

$$
2 A(\infty) c=f(\infty) B(\infty)
$$

are equivalent in the following sense: if $\Phi(z)$ is a general solution for the boundary problem

$$
\Phi^{+}(t)=\frac{A(t)-B(t)}{A(t)+B(t)} \Phi^{-}(t)+\frac{f(t)}{A(t)+B(t)} \quad(-\infty<t<\infty)
$$

satisfying the condition (4.26), where $c$ is the leading coefficient for the polynomial $P_{\chi}(z)$ for $\chi \geq 0$, and $c=-\frac{1}{2 \pi i} \int_{\mathbb{R}} \frac{f(\tau)}{[A(\tau)+B(\tau)] X^{+}(\tau)} \frac{d \tau}{\tau+i}$ for $\chi<0$, then the function $\phi(t)=$ $\Phi^{+}(t)-\Phi^{-}(t)$ is the solution of (4.25). Conversely, if $\phi(t)$ is the general solution to (4.25),

\footnotetext{
${ }^{1}$ Many thanks to Vadim Zharnitsky for aid in the translation.
} 


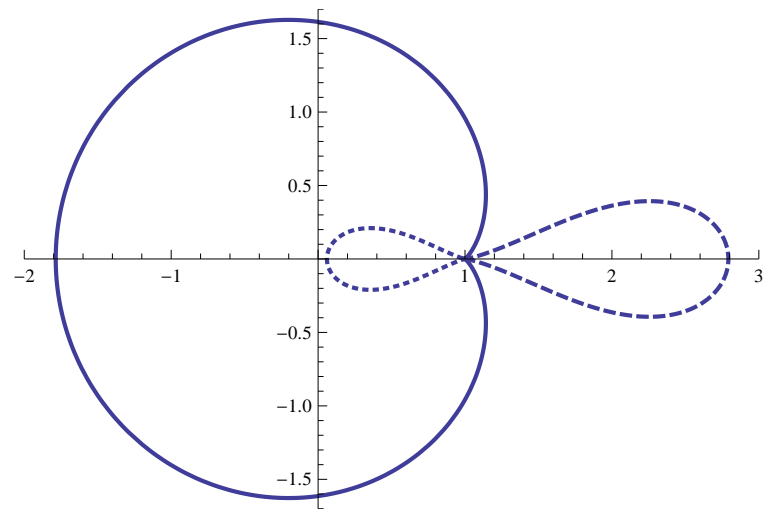

FiguRE 4. Images of the real line under $G_{\xi}(v)$ (solid curve $\xi=1 / 2$, dotted curve $\xi=-2$, dashed curve $\xi=3.75$ )

then the Cauchy type integral $\Phi(z)=\frac{1}{2 \pi i} \int_{\mathbb{R}} \frac{\phi(\tau)}{\tau-z} d \tau$ is the solution of the Riemann problem $(-\infty<t<\infty)$ satisfying the condition $(4.26)$.

In other words, provided the constraint (4.26) is satisfied, the solution to the Riemann problem for the half-plane can be used to construct the solution for (4.21).

4.5.2. Index of the Singular Equation. For our problem,

$$
\chi=\operatorname{Ind} G_{\xi}(v)=\frac{1}{2 \pi}\left[\arg G_{\xi}(v)\right]_{\mathbb{R}} .
$$

is the winding number of the image of the boundary curve $\mathbb{R}$ in $\mathbb{C}$ under the map $G_{\xi}(v)$. Using the definition of $G_{\xi}(v)$, we see that

$$
G_{\xi}(v)=\frac{\xi^{2}-\pi^{2} \phi^{2}(v)+4 D^{2}(v)}{(\xi-\pi \phi(v))^{2}+4 D^{2}(v)}+\left(\frac{4 \pi \phi(v) D(v)}{(\xi-\pi \phi(v))^{2}+4 D^{2}(v)}\right) i
$$

Note that the image of $\mathbb{R}$ is real-valued at only three points, $v=0$ and $v= \pm \infty$. Since $G_{\xi}( \pm \infty)=(1,0)$, every image curve starts and ends at $(1,0)$. At $v=0, G_{\xi}(0)=\left(\frac{\xi+\sqrt{\pi}}{\xi-\sqrt{\pi}}, 0\right)$. For the image curve to have a non-zero winding number, it is necessary that $\frac{\xi+\sqrt{\pi}}{\xi-\sqrt{\pi}}<0$. We find that for $\xi \in(-\sqrt{\pi}, \sqrt{\pi}), \chi=-1$. For all other $\xi, \chi=0$. (For examples, see Figure 4.)

4.5.3. Solution to the Associated Riemann Problem. Let $X^{+}(v)=e^{\Gamma^{+}(v)}$ and $X^{-}(v)=$ $\left(\frac{v-i}{v+i}\right)^{-\chi} e^{\Gamma^{-}(v)}$ where

$$
\Gamma(v)=\frac{1}{2 \pi i} \int_{\mathbb{R}} \ln \left[\left(\frac{\tau-i}{\tau+i}\right)^{-\chi} G_{\xi}(\tau)\right] \frac{d \tau}{\tau-v},
$$

and let

$$
\Psi(v)=\frac{1}{2 \pi i} \int_{\mathbb{R}} \frac{g_{\xi}(\tau)}{X^{+}(\tau)} \frac{d \tau}{\tau-v}
$$


where $G_{\xi}(v)$ and $g_{\xi}(v)$ are defined as in (4.22). Then the unique solution to the Riemann problem for the half-plane is given by

$$
\Phi(v)=X(v) \Psi(v) .
$$

4.5.4. Solving for the Coefficients. Given the above solution, from repeated use of the SokhotskiPlemelj formulas we get a unique representation for the $K_{\xi}(v)$, namely

$$
\begin{aligned}
K_{\xi}(v) & =\Phi^{+}(v)-\Phi^{-}(v), \\
& =X^{+}(v) \Psi^{+}(v)-X^{-}(v) \Psi^{-}(v), \\
& =X^{+}(v)\left(\frac{1}{2} \frac{g_{\xi}(v)}{X^{+}(v)}+\frac{1}{2 \pi i} \int_{\mathbb{R}} \frac{g_{\xi}(\tau)}{X^{+}(\tau)} \frac{d \tau}{\tau-v}\right)-X^{-}(v)\left(-\frac{1}{2} \frac{g_{\xi}(v)}{X^{+}(v)}+\frac{1}{2 \pi i} \int_{\mathbb{R}} \frac{g_{\xi}(\tau)}{X^{+}(\tau)} \frac{d \tau}{\tau-v}\right), \\
& =\frac{g_{\xi}(v)}{2}\left[1+\frac{1}{G_{\xi}(v)}\right]+\frac{X^{+}(v)}{2 \pi i}\left[1-\frac{1}{G_{\xi}(v)}\right] \int_{\mathbb{R}} \frac{g_{\xi}(\tau)}{X^{+}(\tau)} \frac{d \tau}{\tau-v},
\end{aligned}
$$

since $X^{+}(v)=G_{\xi}(v) X^{-}(v)$. Using the definitions of $X^{+}(v), G_{\xi}(v)$ and $g_{\xi}(v)$ yields the following representation:

$$
K_{\xi}(v)=\frac{A_{\xi}(v)}{A_{\xi}^{2}(v)+B^{2}(v)} F_{\xi}(v)-\frac{e^{\Gamma^{+}(v)} B(v)}{A_{\xi}(v)-B(v)} \frac{1}{\pi i} \int_{\mathbb{R}} \frac{F_{\xi}(\tau)}{e^{\Gamma^{+}(\tau)}\left(A_{\xi}(\tau)+B(\tau)\right)} \frac{d \tau}{\tau-v}
$$

where $A_{\xi}(v)=\xi-2 i D(v), B(v)=-\pi \phi(v)$,

$$
F_{\xi}(v)=\phi(v)\left(\hat{f}(\xi, v)-\frac{C_{\Lambda}(\xi)}{1+\Lambda(\xi)+\xi v i}\right),
$$

and

$$
\Gamma^{+}(v)=\frac{1}{2} \ln \left[\left(\frac{v-i}{v+i}\right)^{-\chi} \frac{A_{\xi}(v)-B(v)}{A_{\xi}(v)+B(v)}\right]+\frac{1}{2 \pi i} \int_{\mathbb{R}} \ln \left[\left(\frac{\tau-i}{\tau+i}\right)^{-\chi} \frac{A_{\xi}(\tau)-B(\tau)}{A_{\xi}(\tau)+B(\tau)}\right] \frac{d \tau}{\tau-v}
$$

(Recall that $D(v)$ is Dawson's function.)

Additionally, when $\chi=-1$, we get a unique representation of the coefficient $C_{\Lambda}(\xi)$. Since $B(\infty)=0$ and $A_{\xi}(\infty)=\xi$, the constraint at infinity condition (4.26) yields the additional condition that $c \equiv 0$, or equivalently

$$
\int_{\mathbb{R}} \frac{F_{\xi}(\tau)}{\left[A_{\xi}(\tau)+B(\tau)\right] X^{+}(\tau)} \frac{d \tau}{\tau+i}=0
$$

The definition of $F_{\xi}(v)$ yields

$$
C_{\Lambda}(\xi)=\frac{\int_{\mathbb{R}} \frac{\phi(\tau) \hat{f}(\xi, \tau)}{e^{\Gamma^{+}(\tau)}\left[A_{\xi}(\tau)+B(\tau)\right][1+\Lambda(\xi)+\xi \tau i]} \frac{d \tau}{\tau+i}}{\int_{\mathbb{R}} \frac{\phi(\tau)}{e^{\Gamma^{+}(\tau)}\left[A_{\xi}(\tau)+B(\tau)\right][1+\Lambda(\xi)+\xi \tau i]} \frac{d \tau}{\tau+i}} .
$$

Now, it is important to note that the form of the coefficients are dependent upon $\chi$ and that $\chi$ is dependent upon $\xi$. Recall that when $|\xi| \geq \sqrt{\pi}, \chi=0$. Additionally, the value of $C_{\Lambda}(\xi)$ is dependent upon $\Lambda(\xi)$ and $\Lambda(\xi)$ is only defined for values of $\xi$ in $(-\sqrt{\pi}, \sqrt{\pi})$. In order to make sense of this, in addition to the requirement $\hat{f}(\xi, v)$ be such that $\phi(v)\left(\hat{f}(\xi, v)-\frac{C_{\Lambda}(\xi)}{1+\Lambda(\xi)+\xi v i}\right)$ is Hölder continuous in $v$ for all $\xi$ in $(-\sqrt{\pi}, \sqrt{\pi})$, we 
need the additional condition that $\hat{f}(\xi, v)$ be in the class of functions such that $C_{\Lambda}(\xi) \equiv 0$ when $|\xi| \geq \sqrt{\pi}$. Since we are requiring that $K_{\xi}(v)=0$ at infinity, we are still guaranteed uniqueness of our solution for all $\xi$.

\section{Applying the Spectral Decomposition}

We now apply the properties of our spectral decomposition to find elementary solutions to the original PIDE (1.1). We have transformed the PIDE into

$$
\frac{\partial \hat{f}}{\partial t}(t, \xi, v)=-v i \xi \hat{f}(t, \xi, v)-\hat{f}(t, \xi, v)+\int_{\mathbb{R}} \phi(w) \hat{f}(t, \xi, w) d w
$$

and we write this as

where $L$ is defined to be

$$
\frac{\partial \hat{f}}{\partial t}(t, \xi, v)=L(\hat{f})(t, \xi, v)
$$

$$
L(g)(\xi, v)=-\xi v i g(\xi, v)-g(\xi, v)+\int_{\mathbb{R}} \phi(w) g(\xi, w) d w .
$$

By the transform arising from our spectral decomposition (4.19), we look for solutions of the form

$$
\hat{f}(t, \xi, v)=\frac{C_{\Lambda}(t, \xi)}{1+\Lambda(\xi)+\xi v i}-\text { p.v. } \int_{\mathbb{R}} \frac{K_{\xi}(t, w) d w}{i(w-v)}+\frac{1}{\phi(v)}\left(\xi-\text { p.v. } \int_{\mathbb{R}} \frac{\phi(w)}{i(w-v)} d w\right) K_{\xi}(t, v) .
$$

Then, by Corollaries 7 and 8, (5.1) becomes

$$
\begin{aligned}
\frac{\frac{d C_{\Lambda}}{d t}(t, \xi)-\Lambda(\xi) C_{\Lambda}(t, \xi)}{1+\Lambda(\xi)+\xi v i} & - \text { p.v. } \int_{\mathbb{R}} \frac{\left[\frac{d K_{\xi}}{d t}(t, w)-(-1-\xi w i) K_{\xi}(t, w)\right] d w}{i(w-v)} \\
& +\frac{1}{\phi(v)}\left(\xi-\mathrm{p} \cdot \mathrm{v} \cdot \int_{\mathbb{R}} \frac{\phi(w)}{i(w-v)} d w\right)\left[\frac{d K_{\xi}}{d t}(t, v)-(-1-\xi v i) K_{\xi}(t, v)\right]=0 .
\end{aligned}
$$

By the uniqueness of our spectral representation, this yields the ODEs

$$
\frac{d C_{\Lambda}}{d t}(t, \xi)-\Lambda(\xi) C_{\Lambda}(t, \xi)=0 \text { and } \frac{d K_{\xi}}{d t}(t, v)-(-1-\xi v i) K_{\xi}(t, v)=0
$$

Hence,

$$
C_{\Lambda}(t, \xi)=\widetilde{C}_{\Lambda}(\xi) e^{\Lambda(\xi) t} \text { and } K_{\xi}(t, v)=\widetilde{K}_{\xi}(v) e^{(-1-\xi v i) t}
$$

Theorem 10. Let $f(0, x, v):=f_{0}(x, v)$ represent the initial molecular (number) density of the gas such that $\hat{f}_{0}(\xi, v)$ has compact support on $\xi \in(-\sqrt{\pi}, \sqrt{\pi})$ and such that

$$
F_{0}(\xi, v)=\phi(v)\left(\hat{f}_{0}(\xi, v)-\frac{C_{0}(\xi)}{1+\Lambda(\xi)+\xi v i}\right)
$$

is Hölder continuous in $v$ on $\mathbb{R}$ when $C_{0}(\xi)$ is defined by

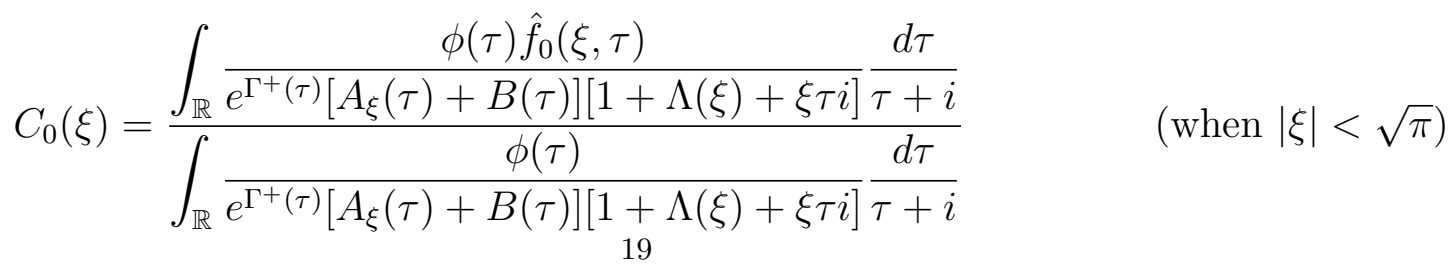


and $C_{0}(\xi) \equiv 0$ when $|\xi| \geq \sqrt{\pi}$ where $A_{\xi}(v)=\xi-2 i D(v), B(v)=-\pi \phi(v)$, and $D(y)=$ $e^{-y^{2}} \int_{0}^{y} e^{x^{2}} d x$ is Dawson's function. Then the Fourier transform of the elementary solution to (1.1) with initial condition is

$$
\begin{aligned}
& \hat{f}(t, \xi, v)=e^{\Lambda(\xi) t} \frac{C_{0}(\xi)}{1+\Lambda(\xi)+\xi v i} \\
&+e^{-t}\left[- \text { p.v. } \int_{\mathbb{R}} \frac{e^{(-\xi w i) t} K_{0}(\xi, w) d w}{i(w-v)}+\frac{e^{(-\xi v i) t}}{\phi(v)}\left(\xi-\text { p.v. } \int_{\mathbb{R}} \frac{\phi(w)}{i(w-v)} d w\right) K_{0}(\xi, v)\right]
\end{aligned}
$$

where

$$
K_{0}(\xi, v)=\frac{A_{\xi}(v)}{A_{\xi}^{2}(v)+B^{2}(v)} F_{0}(\xi, v)-\frac{e^{\Gamma^{+}(v)} B(v)}{A_{\xi}(v)-B(v)} \frac{1}{\pi i} \int_{\mathbb{R}} \frac{F_{0}(\xi, \tau)}{e^{\Gamma^{+}(\tau)}\left(A_{\xi}(\tau)+B(\tau)\right)} \frac{d \tau}{\tau-v}
$$

with

$$
\Gamma^{+}(v)=\frac{1}{2} \ln \left[\left(\frac{v-i}{v+i}\right)^{-\chi} \frac{A_{\xi}(v)-B(v)}{A_{\xi}(v)+B(v)}\right]+\frac{1}{2 \pi i} \int_{\mathbb{R}} \ln \left[\left(\frac{\tau-i}{\tau+i}\right)^{-\chi} \frac{A_{\xi}(\tau)-B(\tau)}{A_{\xi}(\tau)+B(\tau)}\right] \frac{d \tau}{\tau-v}
$$

\section{The elementary solutions evolve to Grossly Determined Solutions}

We are now at a point where we can demonstrate a restricted version of the third conjecture of Truesdell and Muncaster [28]: that elementary solutions should evolve asymptotically in time to the class of grossly determined solutions. At this point, we will see that this amounts to nothing more than definition chasing. What was unexpected apriori is how different portions of the spectrum correspond to the asymptotic behavior of the solution. Roughly speaking, the portion of the elementary solution corresponding to the real-valued part of the spectrum, $(-1,0]$, tends in time specifically to the subclass of solutions defined by the grossly determined solutions; the portion of the elementary solution corresponding to the vertical line $\boldsymbol{\ell}$ in $\mathbb{C}$ is transient.

Recall that $\Lambda(\xi)$ takes values in the open interval $(-1,0)$ (see Figure 2 ). Hence, the asymptotic behavior of the elementary solution (5.2) tends to the part of the spectral decomposition arising from the real-part of the spectrum. In other words,

$$
\hat{f}(t, \xi, v) \sim e^{\Lambda(\xi) t} \frac{C_{0}(\xi)}{1+\Lambda(\xi)+\xi v i} . \quad(\text { as } t \rightarrow \infty)
$$

The main achievement in [2] was to construct the class of grossly determined solutions to (1.1). By ansatz (motivated by a lemma of Hörmander [21, pg 15]), one looked for convolution solutions of the form $f(t, x, v)=\int_{\mathbb{R}} K_{v}(y) \rho(t, x-y) d y$, where $\rho(t, x)$ is the density field. The ultimate goal of this paper was to show that any element from the class of elementary solutions decays (in time) to an element of the subclass of grossly determined solutions. For completeness, we include the one dimensional version of the Main Theorem of $[2]$.

Theorem 11. Consider the one dimensional model of fluid flow

$$
\frac{\partial f}{\partial t}(t, x, v)+v \frac{\partial f}{\partial x}(t, x, v)=-f(t, x, v)+\int_{\mathbb{R}} f(t, x, w) \phi(w) d w
$$


where $f(t, x, v)$ is the molecular density function of the gas and $\phi$ is the probability density function $\phi(v):=e^{-v^{2}} / \sqrt{\pi}$. Let $\rho(t, x)$ represent the density function of the gas:

$$
\rho(t, x):=\int_{\mathbb{R}} f(t, x, v) \phi(v) d v .
$$

Then a solution to equation (6.1) is given by

$$
f(t, x, v)=\int_{\mathbb{R}} K_{v}(y) \rho(t, x-y) d y
$$

where $\rho$ satisfies the associated continuity equation:

$$
\partial_{t} \rho+\partial_{x} M=0
$$

in which $M$ is the mass-flux field

$$
M(t, x)=\int_{\mathbb{R}} v f(t, x, v) \phi(v) d v .
$$

The Fourier transform form of $\rho$ that solves the continuity equation (6.3) is

$$
\hat{\rho}(t, \xi)=\hat{\rho}_{0}(\xi) e^{-i k(\xi) t}
$$

where $\hat{\rho}(t, \xi)$ has support within $|\xi| \in(0, \sqrt{\pi})$ and $\hat{\rho}_{0}(\xi)$ denotes the Fourier transform of the density function at $t=0$.

Then the Fourier transform form of the solution to (6.1) is given by

$$
\hat{f}(t, \xi, v)=\left(\frac{1}{1-k(\xi) i+\xi v i}\right) \hat{\rho}_{0}(\xi) e^{-i k(\xi) t}
$$

where $k(\xi)=(-1+\xi C(\xi)) i$ and $c=C(\xi)$ is defined implicitly by

$$
\xi=\int_{\mathbb{R}} \frac{c \phi(v)}{c^{2}+v^{2}} d v
$$

With just a minor bit of manipulation, we can easily see that the portion of the elementary solution that corresponds to the real part of the spectrum also corresponds to the subclass of grossly determined solutions. Recall that $\Lambda(\xi)$ was defined $\Lambda(\xi)=-1+\xi C(\xi)$. Hence,

$$
-k(\xi) i=k(\xi) \cdot(-i)=-1+\xi C(\xi)=\Lambda(\xi) .
$$

Hence, the grossly determined solution (under transform) (6.6) can be rewritten as

$$
\hat{f}(t, \xi, v)=e^{\Lambda(\xi) t} \frac{\hat{\rho}_{0}(\xi)}{1+\Lambda(\xi)+\xi v i} .
$$

This proves Truesdell and Muncaster's third conjecture for the model Boltzmann (1.1) for elementary solutions: the subclass of grossly determined solutions acts as an attractor set for the class of elementary solutions. That is, in time, all gas flows represented by this elementary solution set act as grossly determined solutions. 


\section{Conclusions}

In the terms of Truesdell and Muncaster's conjectures on grossly determined solutions, we have demonstrated that there exists a class of elementary solutions that decay asymptotically to the subclass of grossly determined solutions. In other words, there exist gas flows such that the asymptotic gas flows will be dictated solely by the initial density field of the gas. As this model Boltzmann equation is a linearization of the one dimensional Boltzmann equation about a maxwellian distribution, this paper is a first step in showing that Truesdell and Muncaster's three conjectures also hold for one dimensional Boltzmann equations with a more robust collision operator and a general class of initial data.

\section{REFERENCES}

[1] Prabhu Lal Bhatnagar, Eugene P Gross, and Max Krook. A model for collision processes in gases. i. small amplitude processes in charged and neutral one-component systems. Physical review, 94(3):511, 1954.

[2] Thomas E. Carty. Grossly determined solutions for a boltzmann-like equation. Kinetic and Related Models (to appear), 10(4).

[3] Thomas E. Carty. Grossly determined solutions for a boltzmann-like equation, http://arxiv.org/abs/1607.08282v1.

[4] KM Case. Elementary solutions of the transport equation and their applications. Annals of Physics, 9(1):1-23, 1960.

[5] Carlo Cercignani. Elementary solutions of the linearized gas-dynamics boltzmann equation and their application to the slip-flow problem. Annals of Physics, 20(2):219-233, 1962.

[6] Carlo Cercignani. Elementary solutions of the linearized gas-dynamics boltzmann equation and their application to the slip-flow problem. Annals of Physics, 20(2):219-233, 1962.

[7] Carlo Cercignani. The method of elementary solutions for kinetic models with velocity-dependent collision frequency. Annals of Physics, 40(3):469-481, 1966.

[8] Carlo Cercignani. Mathematical methods in kinetic theory. Plenum Press, New York, 1969.

[9] Carlo Cercignani. Methods of solution of the linearized boltzmann equation for rarefied gas dynamics. Journal of Quantitative Spectroscopy and Radiative Transfer, 11(6):973-985, 1971.

[10] Carlo Cercignani, Reinhard Illner, and Mario Pulvirenti. The mathematical theory of dilute gases, volume 106 of Applied Mathematical Sciences. Springer-Verlag, New York, 1994.

[11] Carlo Cercignani and Franco Sernagiotto. The method of elementary solutions for time-dependent problems in linearized kinetic theory. Annals of Physics, 30(1):154-167, 1964.

[12] Ch Dalitz. Half-space problem of the boltzmann equation for charged particles. Journal of statistical physics, 88(1-2):129-144, 1997.

[13] EH De Groot and C Dalitz. Exact solution of a boundary value problem in semiconductor kinetic theory. Journal of Mathematical Physics, 38(9):4629-4643, 1997.

[14] Emanuele Dolera. On the computation of the spectrum of the linearized Boltzmann collision operator for Maxwellian molecules. Boll. Unione Mat. Ital. (9), 4(1):47-68, 2011.

[15] Ricardo Estrada and Ram P. Kanwal. Singular integral equations. Birkhäuser Boston Inc., Boston, MA, 2000.

[16] F. D. Gakhov. Boundary value problems. Translation edited by I. N. Sneddon. Pergamon Press, Oxford, 1966.

[17] F. D. Gakhov. Kraevye zadachi. Izdat. "Nauka", Moscow, 1977. Third edition, revised and augmented.

[18] Laurent Gosse. Well-balanced schemes using elementary solutions for linear models of the boltzmann equation in one space dimension. Kinetic and related models, 5(2):283-323, 2012.

[19] R. J. Hangelbroek. Linear analysis and solution of neutron transport problems. Transport Theory Statist. Phys., 5(1):1-85, 1976.

[20] S. Harris. An Introduction to the Theory of the Boltzmann Equation. Dover Books on Physics. Dover Publications, 2012. 
[21] Lars Hörmander. Linear partial differential operators. Springer Verlag, Berlin, 1976.

[22] H. G. Kaper, C. G. Lekkerkerker, and J. Hejtmanek. Spectral methods in linear transport theory, volume 5 of Operator Theory: Advances and Applications. Birkhäuser Verlag, Basel, 1982.

[23] Tomaž Klinc. On completeness of eigenfunctions of the one-speed transport equation. Communications in Mathematical Physics, 41(3):273-279, 1975.

[24] Rainer Kress. Linear integral equations, volume 82 of Applied Mathematical Sciences. Springer-Verlag, Berlin, 1989.

[25] Constanze Liaw and Sergei Treil. Singular integrals, rank one perturbations and clark model in general situation, 2015, arXiv:1506.00072.

[26] N. I. Muskhelishvili. Singular integral equations. Dover Publications Inc., New York, 1992. Boundary problems of function theory and their application to mathematical physics, Translated from the second (1946) Russian edition and with a preface by J. R. M. Radok, Corrected reprint of the 1953 English translation.

[27] Barry Simon. Spectral analysis of rank one perturbations and applications. In CRM Lecture Notes, volume 8, pages 109-149, 1995.

[28] C. Truesdell and R. G. Muncaster. Fundamentals of Maxwell's kinetic theory of a simple monatomic gas, volume 83 of Pure and Applied Mathematics. Academic Press Inc. [Harcourt Brace Jovanovich Publishers], New York, 1980. Treated as a branch of rational mechanics.

[29] J. A. C. Weideman. Computing the Hilbert transform on the real line. Math. Comp., 64(210):745-762, 1995.

Department of Mathematics, Bradley University, Peoria, IL 61625

E-mail address: tcarty@bradley.edu 